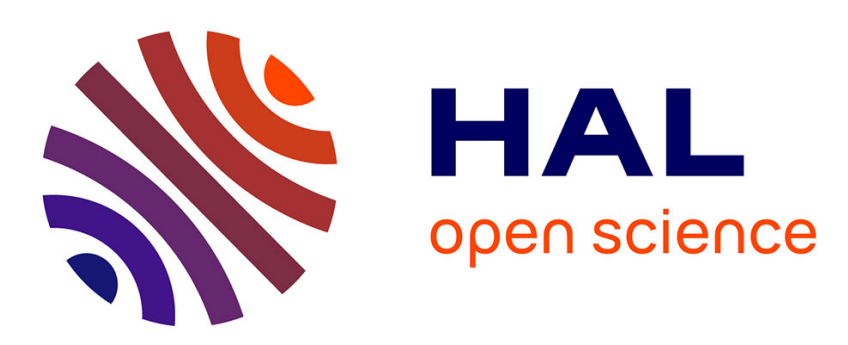

\title{
Détermination au moyen d'un test biologique de la cinétique de libération du phosphore à partir d'une boue résiduaire
}

\author{
Bernard Pommel, M. Lasserre
}

\section{To cite this version:}

Bernard Pommel, M. Lasserre. Détermination au moyen d'un test biologique de la cinétique de libération du phosphore à partir d'une boue résiduaire. Agronomie, 1981, 1 (6), pp.467-472. hal00884282

\section{HAL Id: hal-00884282 \\ https://hal.science/hal-00884282}

Submitted on 1 Jan 1981

HAL is a multi-disciplinary open access archive for the deposit and dissemination of scientific research documents, whether they are published or not. The documents may come from teaching and research institutions in France or abroad, or from public or private research centers.
L'archive ouverte pluridisciplinaire HAL, est destinée au dépôt et à la diffusion de documents scientifiques de niveau recherche, publiés ou non, émanant des établissements d'enseignement et de recherche français ou étrangers, des laboratoires publics ou privés. 


\title{
Détermination au moyen d'un test biologique de la cinétique de libération du phosphore à partir d'une boue résiduaire
}

\author{
Bernard POMMEL \\ avec la collaboration technique de M. LASSERRE, \\ I.N.R.A., Station d'Agronomie, Centre de recherches de Bordeaux, F 33140 Pont de la Maye.
}

RÉSUMÉ

Boue résiduaire,

Phosphore,

Assimilabilité,

Cinétique,

Test biologique,

Ray-grass,

Modèle mathématique.
La cinétique de la libération du phosphore d'une boue résiduaire a été étudiée, à court et moyen termes, grâce à un test biologique : 3 doses de phosphore ont été appliquées, sous forme de boue digérée ou de phosphate monocalcique, dans des pots contenant du sable, ensemencés de ray-grass (Lolium hybridum Hausskn. var. "Sabrina") puis mis en chambre climatisée. Selon la méthode de Stanford \& Dement (1957), les parties aériennes du ray-grass ont été récoltées après 7 jours de contact entre racines et source de $P$. Les cultures ont ensuite été poursuivies pendant 17 cycles successifs, jusqu'à l'épuisement des sources de phosphore, ce qui a requis près de 300 jours.

L'efficience relative du phosphore de la boue a été définie comme le rapport entre les pentes des droites de régression liant l'exportation cumulée de phosphore à l'apport de phosphore, sous forme de boue d'une part et sous forme de phosphate monocalcique d'autre part.

Les variations de cette efficience relative en fonction du temps sont décrites à plus de 99 p. 100 par l'équation suivante :

$$
E=16.6 t^{0.245} \text { ou } \quad E \simeq 16.6 \sqrt{\sqrt{t}}
$$

où E représente l'efficience (en p. 100) et t le temps de contact entre racines et source de phosphore, en jours, avec $20<\mathrm{t}<300$.

L'efficience relative de la boue comme source alimentaire de phosphore augmente progressivement avec le temps : de $17 \mathrm{p} .100$ après $7 \mathrm{j}$, elle s'élève à $50 \mathrm{p} .100$ après $100 \mathrm{j}$ et atteint $66 \mathrm{p} .100$ après $300 \mathrm{j}$. Les boues doivent donc être considérées comme un engrais phosphaté à action lente.

\section{SUMMARY}

Sewage sludge, Phosphorus, Availability,

\section{Kinetics,}

Biological test,

Rye-grass,

Mathematic model.

\section{Study of the kinetics of sewage sludge phosphorus release by a biological test}

The kinetics of sewage sludge phosphorus release was studied by a biological short and middle term test : 3 doses of phosphorus were supplied as digested sewage sludge or as monocalcium phosphate in pots filled with sand and planted with ray-grass (Lolium hybridum Hausskn. var. "Sabrina »), in a growth chamber. According to the STANFORD \& DEMENT method, ray-grass tops were harvested after 7 days of root soil contact ; cultivations were then continued through 17 cuttings until depletion of phosphorus sources, that required nearly 300 days.

The relative efficiency of sludge phosphorus was defined as the ratio between slopes of regression straight lines of cumulative phosphorus uptake to phosphorus supply, as sludge on one hand, or as monocalcium phosphate on the other hand. This relative efficiency as a function of time was described by the following equation that accounts for more than 99 p. 100 of variation :

$$
E=16.6 t^{0.245} \text { or } E \simeq 16.6 \sqrt{\sqrt{t}}
$$

where $\mathrm{E}=$ efficiency (p. 100) $; \mathrm{t}=$ time of root phosphorus source contact, in days, with $20<\mathrm{t}<300$. The relative efficiency of sludge phosphorus increases gradually with time : from 17 p. 100 after 7 days, it climbs up to 50 p. 100 after 100 days and to $66 \mathrm{p} .100$ after about 300 days. Therefore sludge has to be regarded as a slow release phosphorus fertilizer.

\section{INTRODUCTION}

Le traitement des eaux usées dans les stations d'épuration entraîne la séparation par décantation d'un résidu boueux qu'il faut ensuite recycler ou éliminer.
Le contenu de ce produit en azote, phosphore, matière organique... offre la possibilité d'envisager une valorisation en agriculture.

Contrairement à l'azote et aux métaux lourds, le phosphore des boues n'a été encore que peu étudié (GUPTA, 
1976 ; MOREL, 1977 ; FARDEAU et al., 1977). Il trouve soǹ origine dans les détergents pour 60 p. 100 environ, et dans les matières fécales pour 40 p. 100 (WERnER, 1976). Parmi 189 échantillons de ce produit, la valeur moyenne de la teneur en P trouvée par SOMMERS (1977) était de 2,5 p. 100 (rapportée à la matière sèche) et les limites extrêmes: $<0,1-14,3$ p. 100 . La majeure partie de ce phosphore se trouve dans la phase solide et sous forme minérale (SOMMERS et al., 1976; HANOTIAUX et al., 1980).

Pour suivre les variations du flux de P offert par la boue, nous avons étudié pendant $300 \mathrm{j}$ la cinétique de l'absorption du P par le ray-grass (Lolium hybridum Hausskn. var. "Sabrina »), en utilisant le phosphate monocalcique comme source de référence.

\section{MATÉRIEL ET MÉTHODES}

La boue résiduaire utilisée (tabl. 1) provient d'une station d'épuration de la banlieue bordelaise ; elle a été digérée en anaérobiose, floculée aux polyélectrolytes puis centrifugée. Au laboratoire, elle a été séchée à $50^{\circ} \mathrm{C}$ et broyée pour améliorer l'homogénéité des prises d'essai et permettre un mélange intime avec le sable; son $\mathrm{pH}$ était de 7,2.

\section{TABLEAU 1}

Composition de la boue étudiée, rapportée à la matière sèche Composition of the sewage sludge (dry matter basis)

\begin{tabular}{lc}
\hline \hline & \\
Cendres $\%$ & 54.4 \\
Carbone \% & 29.2 \\
Azote Kjeldahl \% & 2.79 \\
Phosphore total $\%$ & 2.14 \\
Phosphore minéral \% & 1.75 \\
Calcium $\%$ & 5.4 \\
Magnésium $\%$ & 4.73 \\
Fer \% & 4.72 \\
Cuivre ppm & 309 \\
Manganèse ppm & 9253 \\
Zinc ppm & 7056 \\
Cadmium ppm & 33 \\
Chrome ppm & 74 \\
Nickel ppm & 246 \\
Plomb ppm & 1313 \\
\hline
\end{tabular}

$2 \mathrm{~g}$ de graines de ray-grass hybride tétraploïde "Sabrina » ont été semés dans des pots sans fond contenant $700 \mathrm{~g}$ de sable. Ces pots ont reçu les solutions nutritives dépourvues de phosphore, utilisées par LEMAIRE (1977). Les oligoéléments ont été apportés au moyen d'une solution commerciale contenant en quantités équilibrées et largement suffisantes les éléments : molybdène, bore, manganèse, cuivre, zinc et fer.

Après $20 \mathrm{j}$ de croissance, une semelle de racines se développe à la base du pot; la teneur en phosphore des feuilles du ray-grass n'est alors que de 0,14 en p. 100 de la M.S. Selon la méthode de STANFORD \& DEMENT (1957), la semelle des racines avec les plantes intactes est alors placée au contact des pots inférieurs. Ces pots inférieurs contiennent $700 \mathrm{~g}$ de sable, auxquels a été ajoutée la source de phosphore, variant selon 7 traitements, chacun étant répété 4 fois.

\section{Les 7 traitements sont les suivants:}

$\mathrm{T}_{0}$ : témoin sans apport de phosphore ;

$\mathrm{B}_{1}, \mathrm{~B}_{3}, \mathrm{~B}_{9}$ : boue apportée à raison de $0,58 \mathrm{~g} ; 1,75 \mathrm{~g}$ et 5,25 g par pot, ce qui correspondrait, du point de vue du rapport poids de boue/poids de sable, à des épandages de $3,3 \mathrm{t}, 10 \mathrm{t}$ et $30 \mathrm{t} / \mathrm{ha}$ (sur la base de 4000 t de sable par ha);

$T_{1}, T_{3}, T_{9}$ : le phosphate monocalcique constitue la source de phosphore ; cet élément est apporté en même quantité qu'avec les boues.

De plus, un pot a reçu une quantité pléthorique de phosphore en vue de donner la production maximale de matière sèche, ce qui permet de s'assurer à tout moment que la production de tous les traitements est bien limitée par la fourniture de phosphore et non par une autre déficience ou une toxicité.

Selon CHAusSOD (1978), 1/3 environ de l'azote contenu dans les boues digérées serait rapidement assimilable ; il en a été tenu compte lors de l'apport initial d'azote, de façon à obtenir la même quantité d'azote assimilable dans tous les traitements. Cette exception mise à part, les pots inférieurs ont reçu les mêmes solutions nutritives dépourvues de phosphore que les pots supérieurs. L'apport d'eau est réalisé au moyen d'une mèche qui tapisse le fond des pots inférieurs et dont les extrémités plongent dans un réservoir d'eau : il y a donc 3 pots superposés (QUEMENER, 1968).

L'expérimentation a été conduite en chambre climatisée, à $25^{\circ} \mathrm{C}, 70 \mathrm{p} .100$ d'humidité, avec $16 \mathrm{~h}$ d'éclairement par jour ( 85 watts $/ \mathrm{m}^{2}$, tubes fluorescents blanc industrie).

Les parties aériennes du ray-grass sont récoltées à $3 \mathrm{~cm}$ au-dessus du sol, $7 \mathrm{j}$ après le transfert des pots supérieurs sur les pots inférieurs, puis tous les $15 \mathrm{j}$ jusqu'à arrêt de la croissance, qui survient lorsque la teneur en $P$ de la plante s'est abaissée jusqu'à 0,8 ou 0,7 p. 1000 de la matière sèche - ce qui requiert près de $300 \mathrm{j}$ pour les traitements ayant reçu la dose la plus élevée. Le pot recevant une quantité pléthorique de $\mathrm{P}$ exprime encore à cette date une croissance importante.

A la fin de l'expérience, le $\mathrm{pH}$ moyen des substrats était de 5,8 pour les traitements avec boues, de 5,9 pour les traitements avec le phosphate monocalcique et de 6,3 pour le traitement sans phosphore.

Les parties aériennes des ray-grass ont été séchées à $105^{\circ} \mathrm{C}$, pesées, broyées et la poudre obtenue a été minéralisée par voie sèche pour permettre une détermination des teneurs en phosphore par colorimétrie du complexe phospho-molybdique réduit par l'acide ascorbique.

Les racines et collets ont été séparés du sable, à la main, sur tamis, séchés à $105^{\circ} \mathrm{C}$, pesés, broyés et la poudre obtenue a été minéralisée par voie sèche; l'insoluble chlorhydrique a été recueilli, séché et pesé, ce qui a permis de prendre en compte le poids des grains de sable qui sont inévitablement restés liés au système racinaire. Le phosphore a été dosé par la méthode déjà indiquée.

\section{RESULTATS ET DISCUSSION}

Les exportations de phosphore par les parties aériennes du ray-grass après 7 jours de contact entre les racines et les sources de $\mathrm{P}$ sont indiquées dans la figure 1 . Les points correspondant aux traitements $T_{0}, T_{1}, T_{3}$ d'une part, et $T_{0}$, $B_{1}, B_{3}$ d'autre part, sont presque alignés : les coefficients de corrélation linéaire sont respectivement de 0,99996 et 0,991 . La pente de la $1^{\text {re }}$ droite est significativement non nulle (à 1 p. 1000 ), la $2^{\mathrm{e}}$ ne l'est pas à 5 p. 100 (mais elle le serait à 10 p. 100). Ces pentes sont significativement (à 1 p. 1000 ) différentes l'une de l'autre et leur rapport (boue sur phosphate monocalcique) est de 17 p. 100. Le traitement $\mathrm{T}_{9}$ a reçu une quantité de phosphore soluble qui est pléthorique en comparaison de la capacité d'absorption du 


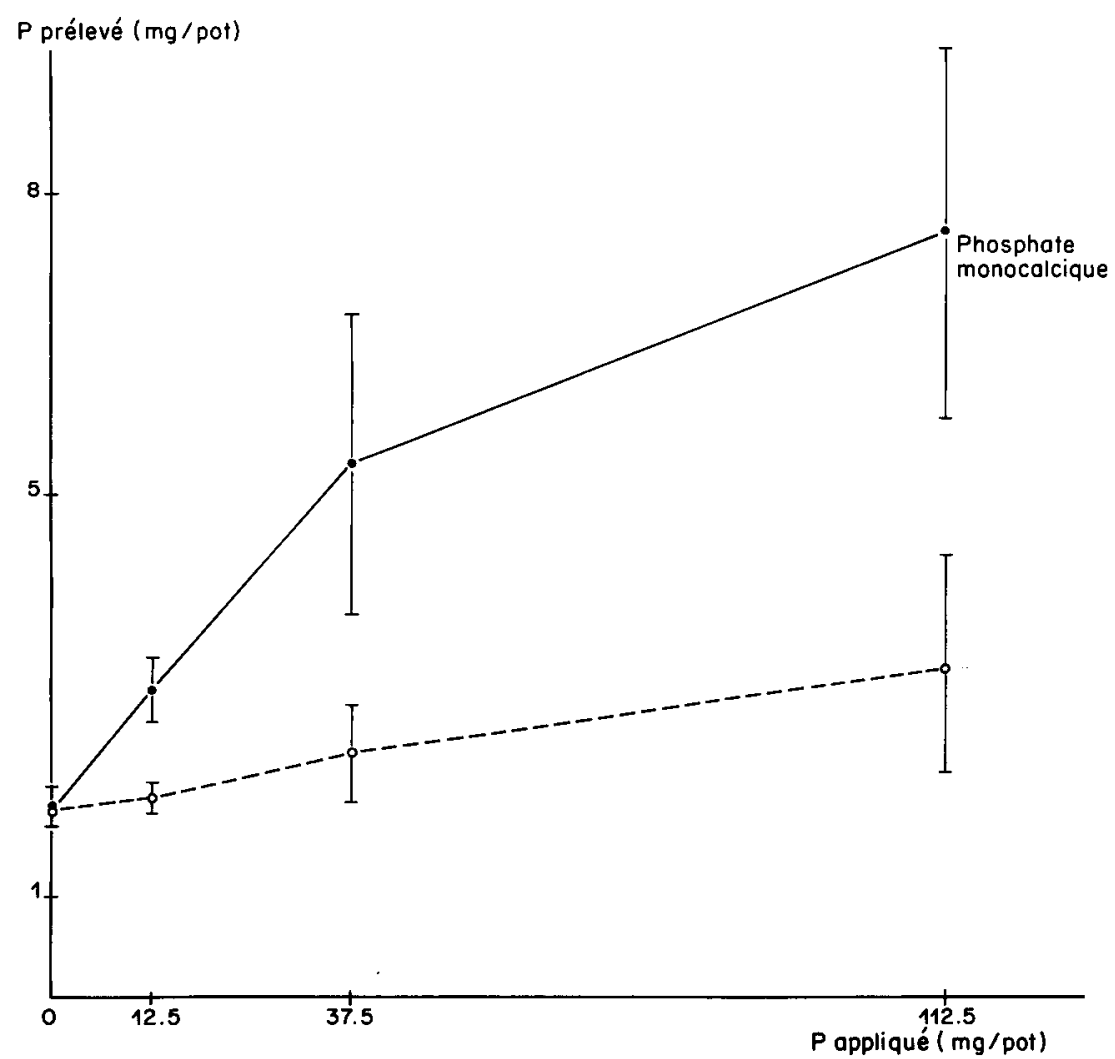

Figure 1

Exportation de phosphore par les parties aériennes du ray-grass après 7 j de contact entre racines et sources de $P$, en fonction de la forme et de la dose d'apport. Intervalles de confiance à 5 p. 100.

Uptake of phosphorus by ray-grass tops after 7 days of root phosphorus source contact, as a function of form and dose of supply. Confidence limits at 5 p. 100.

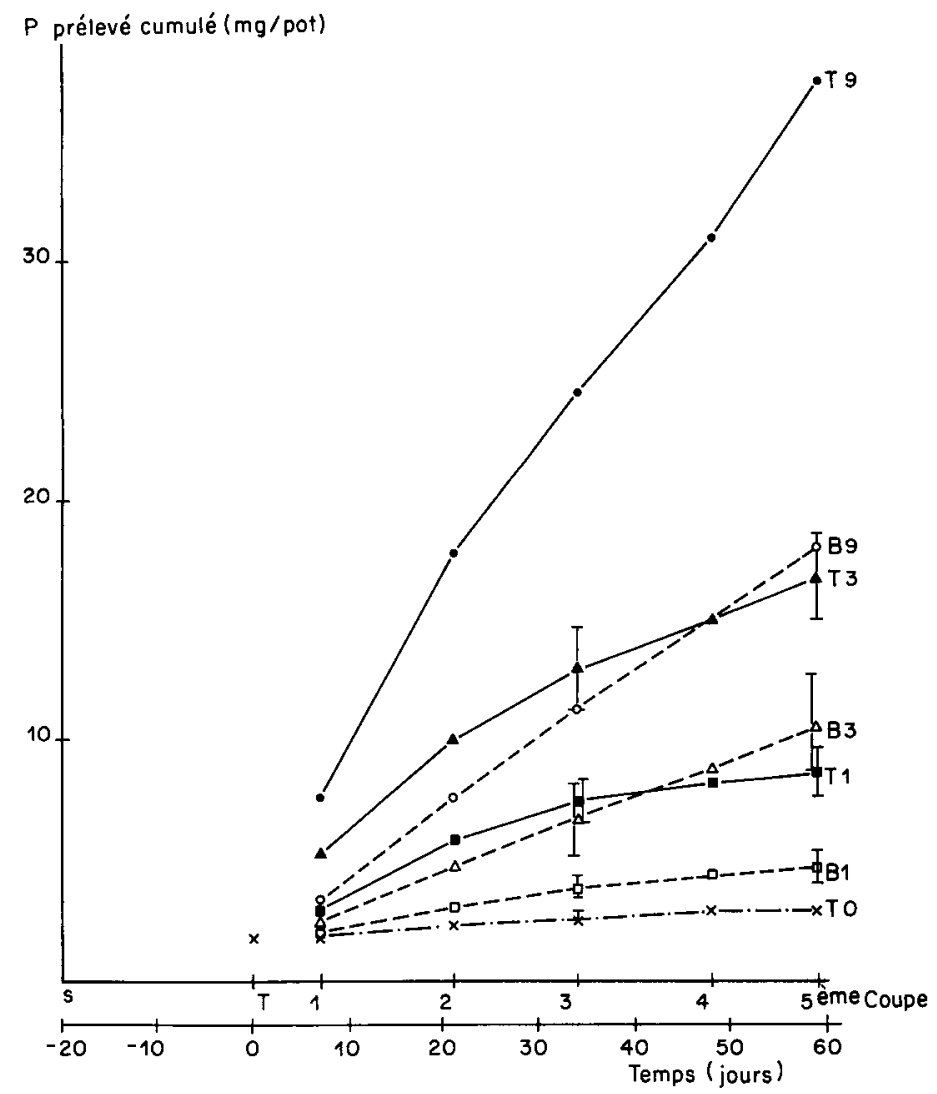

Figure 2

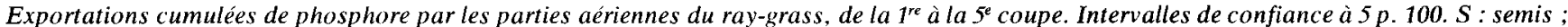
T: transfert.

Cumulative uptakes of phosphorus by ray-grass tops from the 1st to the 5 th cutting. Confidence limits at $5 p .100 . S:$ sowing $; T:$ transference. 


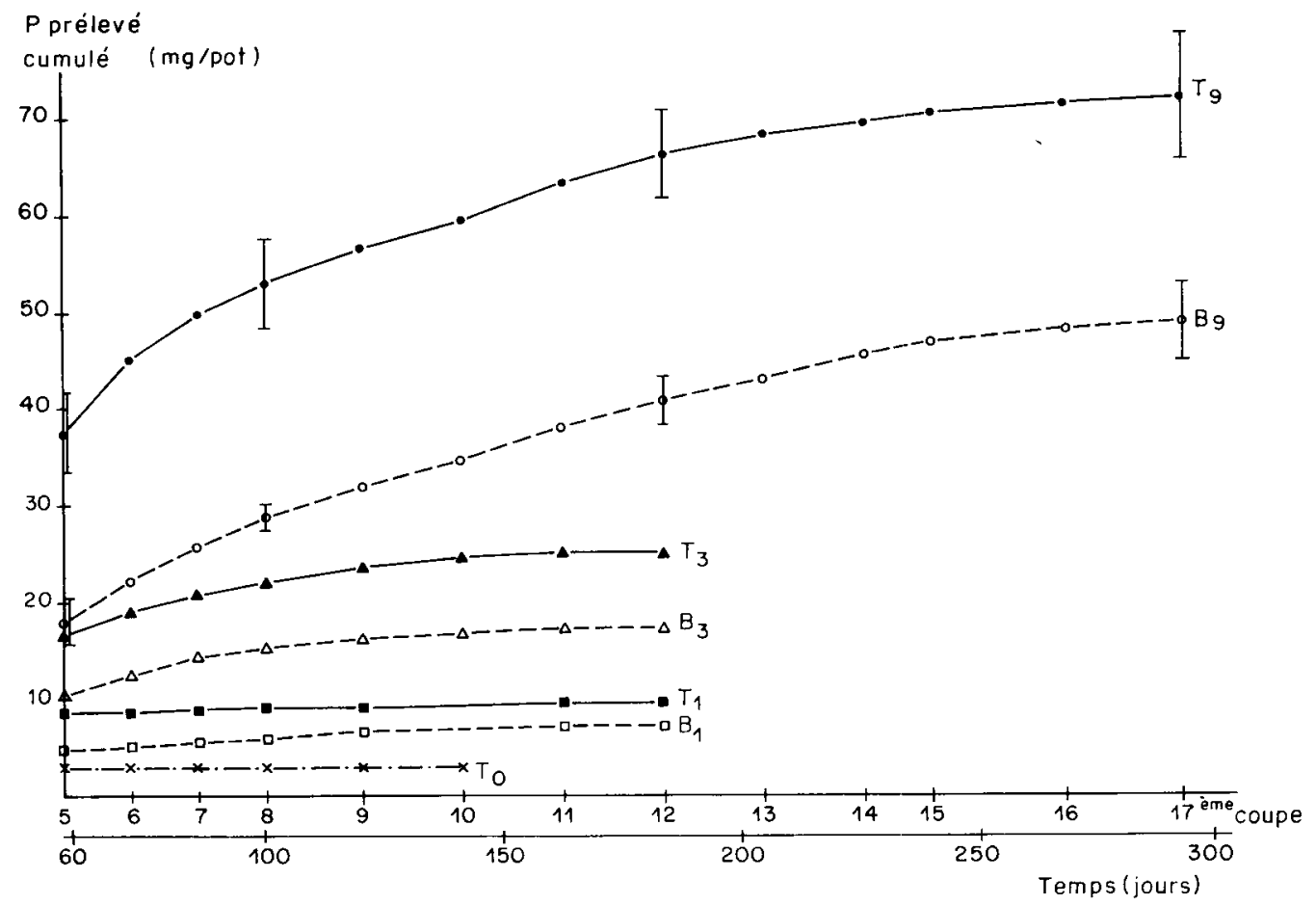

Figure 3

Exportations cumulées de phosphore par les parties aériennes du ray-grass, depuis la $5^{e}$ coupe jusqu'à l'épuisement des sources de P. Intervalles de confiance à 5 p. 100.

Cumulative uptakes of phosphorus by ray-grass tops from the 5th cutting until depletion of $P$ sources. Confidence limits at 5 p. 100 .

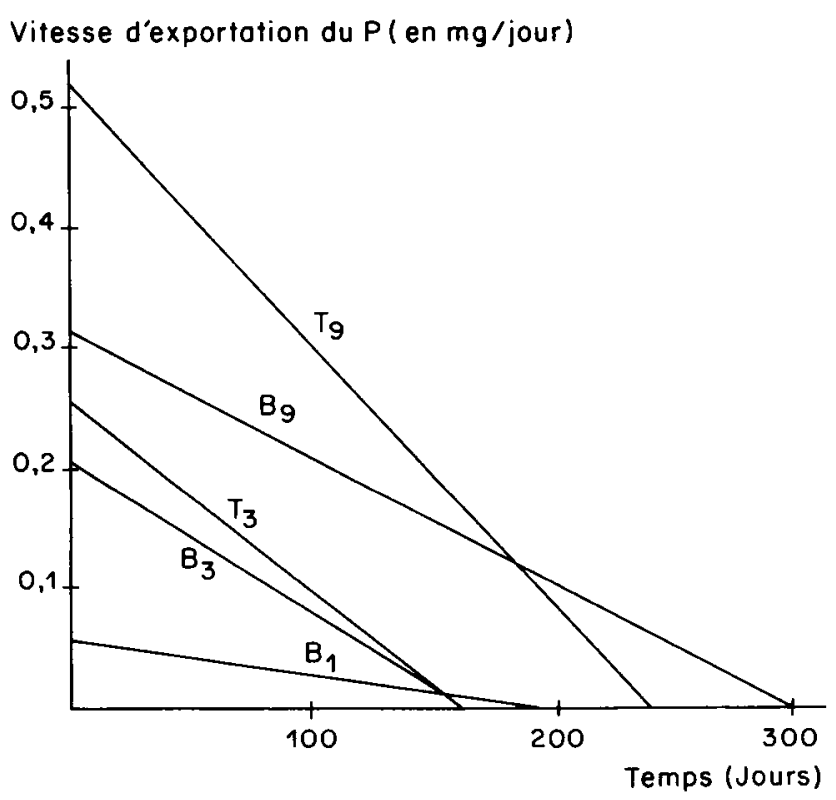

Figure 4

Vitesse d'exportation du phosphore par les parties aériennes du ray-grass, selon les traitements et en fonction du temps.

Speed of $P$ uptake by ray-grass tops, according to treatments, as a function of time.

ray-grass pendant une semaine, aussi la valeur correspondante n'a pas été prise en compte ici mais elle le sera lors des coupes ultérieures. Ainsi, dans le très court terme, la boue n'est capable de fournir au ray-grass qu'une très petite quantité de phosphore.

Les évolutions des exportations cumulées de $\mathrm{P}$ par les parties aériennes du ray-grass sont indiquées dans les figures 2 et 3 . Elles peuvent s'exprimer par des fonctions du second degré du temps (avec $R^{2}>0,985$ ), sauf dans le cas des traitements $T_{0}$ et $T_{1}$. Les vitesses de prélèvement du phosphore sont donc des fonctions linéaires du temps, du type :

$$
\text { vitesse de prélèvement }=a-b t
$$

$a$ et $b$ étant des coefficients positifs (fig. 4). Pour chaque dose d'apport, a et b sont plus élevés dans le cas du phosphate monocalcique que dans celui de la boue. La $1^{\text {ère }}$ source procure donc une meilleure intensité d'alimentation, qui engendre son épuisement plus rapide et un ralentissement plus important de la vitesse de prélèvement.

Les droites de régression des prélèvements cumulés de phosphore rapportés aux apports de cet élément pour les traitements $\mathrm{T}_{0}, \mathrm{~T}_{1}, \mathrm{~T}_{3}, \mathrm{~T}_{9}$ d'une part, et $\mathrm{T}_{0}, \mathrm{~B}_{1}, \mathrm{~B}_{3}, \mathrm{~B}_{9}$ d'autre part, ont été calculées pour chaque coupe de la $2^{\mathrm{e}}$ à la $17^{\mathrm{e}}$. Les coefficients de corrélation linéaire ont toujours été égaux ou supérieurs à 0,98 et étaient supérieurs à 0,9999 pour la $15^{\mathrm{e}}$ coupe (fig. 5). Ils étaient toujours significatifs (à 5 p. 100).

Par comparaison au phosphate monocalcique, l'efficience relative de la boue comme source alimentaire de $\mathrm{P}$ est déterminée par le rapport des pentes des droites de régression concernant les 2 sources. Ces pentes étaient significativement différentes (à 1 p. 1000) pour chaque coupe.

L'efficience relative exprimée en pourcentage de l'effet du phosphate monocalcique a été représentée en fonction du temps dans la figure 6 . Elle varie de la $2^{c}$ à la dernière $*^{*}$ coupe selon l'équation suivante :

$$
E=16.6 t^{0.245} \text { avec } R^{2}>0.99
$$

où $\mathrm{E}=$ efficience relative en $\mathrm{p} .100$ et $\mathrm{t}=$ temps de contact entre racines et source de $P$, en jours, avec $20<t<300$.

L'équation ci-dessus peut encore s'écrire, de façon approximative :

$$
E \simeq 16.6 \sqrt{\sqrt{t}}
$$




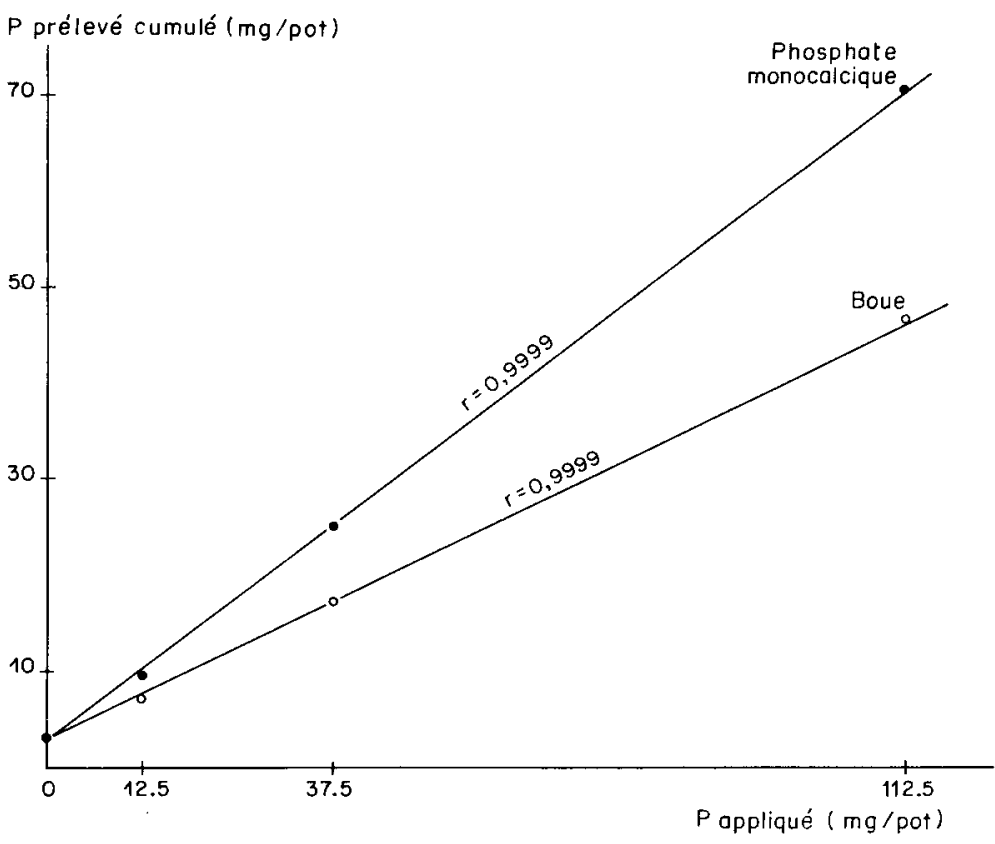

Figure 5

Exportations cumulées de phosphore par les 15 premières coupes de ray-grass, en fonction de la forme et de la dose d'apport. Cumulative uptakes of phosphorus by the first 15 cuttings of ray-grass, as a function of form and dose of supply.

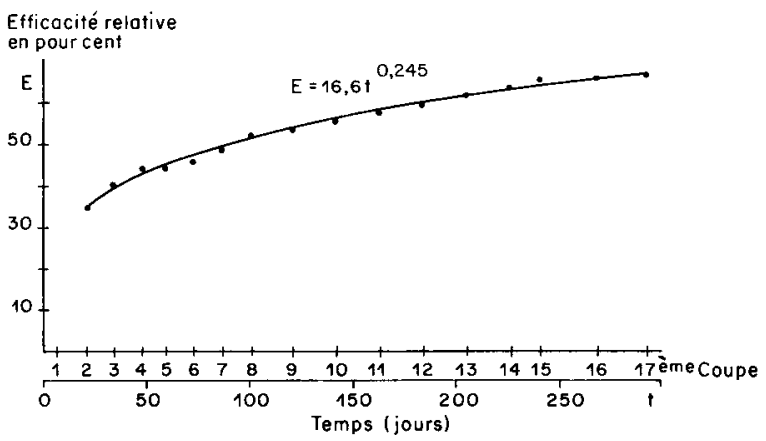

Figure 6

Efficience relative de la boue comme source alimentaire de phosphore exprimée en pourcentage de l'effet du phosphate monocalcique et représentée en fonction du temps.

Percent of relative efficiency of sludge phosphorus compared with monocalcium phosphate phosphorus as a function of time.

expression facile à calculer sur n'importe quelle machine.

L'efficience relative du phosphore de la boue, telle qu'on l'observe lors de la $1^{\text {ère }}$ coupe (pour $t=7$ ), est inférieure à la valeur que laisserait prévoir l'équation ci-dessus. Cette constatation s'explique si l'on invoque une moindre mobilité du phosphore rapidement assimilable de la boue, en grande partie sous forme échangeable, comparativement au phosphore soluble du phosphate monocalcique. En effet, au cours des tout premiers stades de la croissance, la densité racinaire est encore faible et le contact entre racines et source de phosphore n'est pas aussi intime que par la suite. Il faut en conclure que le test de STANFORD \& DEMENT rend compte non seulement d'un facteur d'assimilabilité mais aussi, et peut-être surtout, d'un facteur de mobilité - ce qui rejoint l'opinion d'ALLEN (comm. pers., 1979).

Par ailleurs, dans la phase initiale de la culture, l'effet perturbateur de certains phénomènes est particulièrement accentué : le phosphore contenu dans les graines qui alimente la plante pendant la phase de culture carencée, s'additionne à celui fourni par la boue ou par l'engrais ; d'autre part, la constitution des racines et des collets détourne une partie non négligeable du phosphore prélevé, qui ne sera pas exporté par les coupes des parties aériennes.

Les cultures ont été conduites jusqu'à l'épuisement des sources de P. Après environ $300 \mathrm{j}$, l'efficience relative de la boue se situe à 66 p. 100 et elle n'augmente plus que très lentement. Les coefficients de variation des prélèvements cumulés de $P$ étaient alors de 5 p. 100 sur le traitement $B_{9}$ et

TABLEAU 2

Récapitulation des apports et des prélèvements de phosphore selon les différents traitements Recapitulation of phosphorus supplies and uptakes according to different treatments

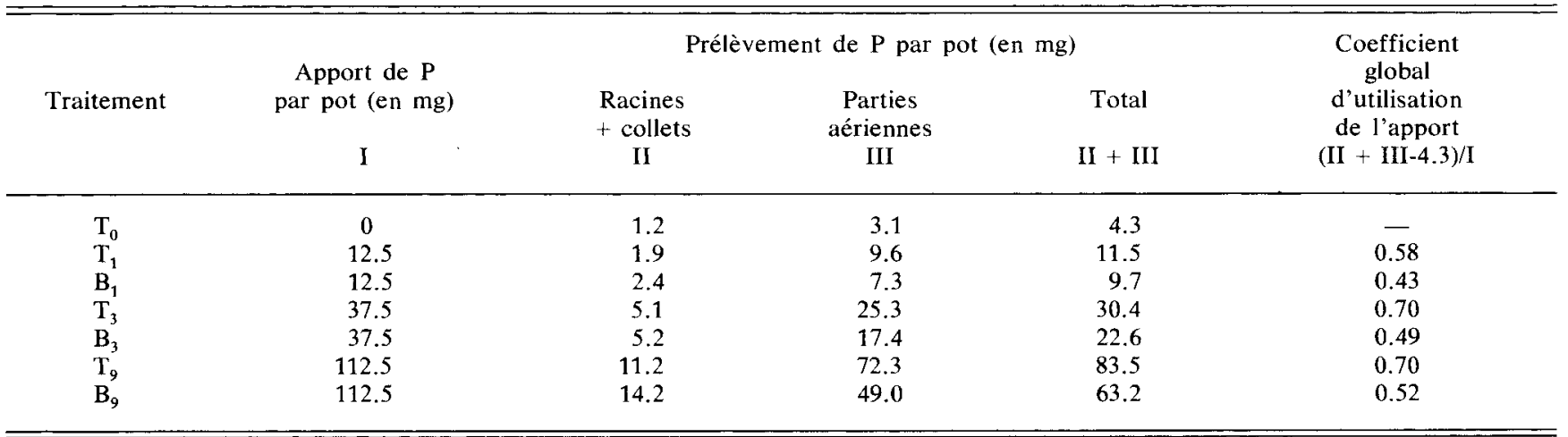


5,8 p. 100 sur le traitement $\mathrm{T}_{9}$. On peut rapprocher de ces résultats ceux de dosages chimiques : à partir de la même boue, le réactif de DYER (1894) a extrait 82 p. $100 \mathrm{du}$ phosphore total, celui de TRUOG (1930) 47 p. 100 et le chlorure de calcium $(0,01 \mathrm{M})$ moins de 1 p. 100 .

Le tableau 2 récapitule les apports et les prélèvements de phosphore.

\section{CONCLUSION}

Initialement, le ray-grass absorbe considérablement plus de $\mathrm{P}$ à partir du phosphate monocalcique que de la boue, en raison de la meilleure intensité d'alimentation que procure la $1^{\text {ère }}$ source. Il en résulte un épuisement plus rapide de“cette source et, par voie de conséquence, l'efficience relative de la boue augmente avec le temps : de 17 p. 100 après 7 j, elle s'élève à 50 p. 100 après $100 \mathrm{j}$ et atteint $66 \mathrm{p} .100$ après $300 \mathrm{j}$. Il faut donc considérer la boue comme un engrais phosphaté à action lente.

Le test utilisé ci-dessus a permis de modéliser, au moyen d'une loi mathématique simple, le comportement intrinsèque d'une boue mise en contact avec un système racinaire dans des conditions climatiques et nutritionnelles contrôlées, le sable utilisé comme substrat ne jouant que le rôle de support inerte. Il est certain que le remplacement du sable par un sol agricole introduirait une complication supplémentaire: le pouvoir fixateur intervient alors en concurrent pour la plante et les termes de la comparaison entre boue et engrais sont changés, car on peut penser qu'en présence d'une boue élevant moins brutalement la concentration de la solution du sol, on limite les phénomènes de fixation.

Par ailleurs, il n'est sans doute pas possible d'extrapoler directement dans les conditions du champ des résultats obtenus en vases de végétation car le rapport de la surface des racines au volume de sol exploré est très différent dans ces 2 situations.

Dans les sols normalement pourvus en phosphore, les cultures n'utilisent annuellement que 5 à $10 \mathrm{p}$. $100 \mathrm{de}$ la réserve de phosphore assimilable et le but de la fertilisation phosphatée annuelle réside plus dans le maintien de la fertilité initiale du sol que dans la fourniture de $\mathrm{P}$ à la culture à venir (GACHON, 1977). En ce sens, on pourrait penser maintenir la fertilité du sol en appliquant des boues, mais il reste nécessaire de s’interroger sur l'évolution à long terme dans le sol des fractions phosphatées de la boue.

D'autres éléments sont à considérer quand on cherche à apprécier la valeur fertilisante du phosphore des boues : dans un essai au champ, JUSTE \& SOLDA (1980) observent que des applications de boue augmentent le contenu du sol en phosphore extractible à l'acide citrique mais n'élèvent pas la concentration du phosphore dans les feuilles de maïs comme le font de bien moindres apports de superphosphate.

Ceci pourrait s'expliquer par la présence, dans les boues, de métaux comme le zinc, qui jouent un rôle antagoniste dans l'absorption du phosphore par la plante (POMMEL, 1979) ; par ailleurs, les métaux lourds limitent la croissance des radicelles, ce qui a pour effet de diminuer le volume de sol exploré et peut induire une déficience en $\mathrm{P}$ dans les plantes (Foy et al., 1978).

Enfin, il ne faut pas oublier que certains traitements appliqués aux boues (par exemple, avec du chlorure ferrique ou du sulfate d'alumine et de la chaux) peuvent modifier l'assimilabilité de leur phosphore (FURRER, 1977 ; CHAWLA et al., 1974). FARDEAU et al. (1979) ont même observé une rétrogradation du phosphore assimilable du sol consécutive à l'apport de boues conditionnées par de la chaux.

\section{Reçu le 15 novembre 1980 Accepté le 6 mars 1981}

\section{RÉFÉRENCES BIBLIOGRAPHIQUES}

Chaussod R., 1978. Valeur fertilisante des boues résiduaires. Les problèmes liés à l'utilisation de l'azote. Cycle Formation permanente, 18-20/4/78, E.N.S.A.I.A., Nancy.

Chawla V. K., Bryant D. N., Liu D., 1974. Disposal of chemical sewage sludges on land and their effects on plants, leachate and soil systems. Sludge handling and disposal seminar. Conf. Proc. $\mathrm{n}^{\circ} 2$, 207-233., Ontario Min. Environnement, Toronto.

Dyer B., 1894. Détermination des matières minérales assimilables par les plantes. Ann. agron., 291-298.

Fardeau J. C., Guiraud G., Hétier J. M., 1977. Répercussions de l'épandage de boues résiduaires sur la mobilité de $\mathrm{Cd}, \mathrm{Cr}, \mathrm{Hg}, \mathrm{Zn}$ et $\mathrm{P}$ dans les sols. $1^{\text {er }}$ Symposium sur la recherche en matière de sols et déchets solides. Orléans-La Source, 15-17/3/77. Collection Recherche-Environnement $\mathrm{n}^{\circ} 11$.

Fardeau J. C., Hétier J. M., Guiraud G., 1979. Étude au moyen de ${ }^{15} \mathrm{~N},{ }^{32} \mathrm{P},{ }^{65} \mathrm{Zn},{ }^{109} \mathrm{Cd}$ et ${ }^{203} \mathrm{Hg}$ de quelques limites d'utilisation en agriculture des boues résiduaires. Symposium européen sur la caractérisation et l'utilisation des boues résiduaires, Cadarache, 13-15/2/79.

Foy C. D., Chaney R. L., White M. C., 1978. The physiology of metal toxicity in plants. Ann. Rev. Plant Physiol. 29, 511-566.

Furrer O. J., 1977. Der Dungerwert des Klärschlammes. Klärschlamm-Verwertung in der Landwirtschaft. Schweig. Landu: Technikum, Zollikofen, 3 mars, 14 p.

Gachon L., 1977. Utilité d'un bon niveau de réserves phosphatées du sol. Phosphore et Agriculture 70, 27-33.

Gupta S. K., 1976. Phosphate removal in systems $\mathrm{H}_{3} \mathrm{PO}_{4}-\gamma-$ $\mathrm{FeO}(\mathrm{OH})$ and $\mathrm{H}_{3} \mathrm{PO}_{4}-\mathrm{FeCl}_{3}$ and characteristics of sludge phosphate. Thesis, Faculty of natural sciences, University of Berne, $138 \mathrm{p}$.

Hanotiaux G., Heck J. P., Rocher M., Barideau L., Marlier-Geets O., 1980. The content of $\mathrm{P}$ in the sewage of Wallonian water purification plants and the form of $\mathrm{P}$ in these. Seminar on phosphorus in sewage sludge and in animal waste, 12-13 June, Haren (Gr). The Netherlands.

Juste C., Solda P., 1980. Effets de l'application massive de boue à très forte charge en cadmium et en nickel sur des cultures de maïs et de laitue. E.E.C. Symp, on characterization, treatment and use of sewage sludge, Vienna, 21-24 October.

Lemaire F., 1977. Nouvelles observations sur l'appréciation de la fertilité des sols cultivés au moyen de l'expérimentation en petits vases de végétation. Ann. agron. 28, 425-444.

Morel J. L., 1977. Contribution à l'étude de l'évolution des boues résiduaires dans le sol. Thèse, Université de Nancy I, $117 \mathrm{p}$.

Pommel B., 1979. Comparaison de l'utilisation par le maîs du phosphore des boues résiduaires ou du phosphate monocalcique pour différents niveaux de zinc et de fer dans le substrat. Ann. agron. 30, 443-45.

Quemener J., 1968. Exemples d'application de la méthode de Stanford Dement à des problèmes de fertilisation phosphatée. Ann. agron. 19, 589-604.

Sommers L. E., 1977. Chemical composition of sewage sludges and analysis of their potential use as fertilizers. J. Environ. Qual. 6, 225-232.

Sommers L. E., Nelson D. W., Yost K. L., 1976. Variable nature of chemical composition of sewage sludges. J. Environ. Qual. 5, 303-306.

Stanford G., Dement J. D., 1957. A method for measuring short term nutrient absorption by plants. I : Phosphorus. Soil Sci., Amer. Proc. 21, 612-617.

Truog E., 1930. The determination of the readily available phosphorus of soil. J. amer. Soc. Agron., 874-882.

Werner W., 1976. Untersuchungen zur Phosphatwirkung von Klärsschlämmen aus der chemischen Ab-wasserreinigung. Landwirtsch. Forsch. 32, 177-185. 